\title{
Receding-horizon pseudo-spectral control of wave energy converters using periodic basis functions
}

\author{
Clément Auger, Alexis Mérigaud and John V. Ringwood, Senior Member, IEEE,
}

\begin{abstract}
Power-maximising control of wave energy converters (WECs) can be implemented within a receding-horizon (RH) framework, whereby the reference force or velocity trajectory is updated in real-time by a feed-forward control (FFC) algorithm. For the computation of optimal WEC trajectories and control forces, Fourier pseudo-spectral (PS) control, using periodic basis functions, is simple and computationally attractive, for both linear and non-linear WEC dynamics. However, the finite-length wave excitation signal, as seen by the FFC, is in general nonperiodic. In this paper, it is shown that, despite the nonperiodicity of the input wave signal, a simple Fourier PS method can be successfully used as a FFC algorithm, by applying suitable windowing functions to the input signal when necessary. Furthermore, a simple wave forecasting algorithm is introduced, solely based on past values of the wave excitation, and the impact of wave forecast errors on the controller performance is investigated. Overall, with or without forecast errors, the proposed approach allows for power absorption above $99 \%$ of the optimum, for a forecasting horizon of less than $\mathbf{3 0}$ seconds for a linear WEC model, and less than 15 seconds for a non-linear WEC model including a quadratic viscous drag term.
\end{abstract}

Index Terms-Wave energy converters, optimal control, power maximisation, receding-horizon control, Fourier pseudo-spectral methods, wave forecasting

\section{INTRODUCTION}

$\mathbf{P}$ OWER-MAXIMISING control has the potential to significantly improve the economic competitiveness of wave energy converters (WECs) [1]. Due, in particular, to radiation force memory effects, the optimal control law for WEC power maximisation is, in general, non-causal, i.e. the knowledge of future wave excitation is required [2]. Thus, $\mathrm{RH}$, modelpredictive control provides a relevant framework which utilises wave excitation forecasts over a finite time horizon, where the optimal trajectory (for device velocity or control force) is updated in real time, as new wave input forecasts become available [3]. The general receding-horizon WEC control philosophy is illustrated in Fig. 1, showing the reference WEC velocity (optimal velocity prediction) updated at two consecutive time steps. For a thorough overview of modelpredictive WEC control algorithms (implemented or not in a RH fashion), the reader is referred to [4].

In this paper, the control structure is assumed hierarchical, as in [5]. The optimal trajectory is updated through a feedforward calculation (from the feed-forward controller, FFC) which is, in fact, an optimisation problem [1], whereby power

C. Auger is with ENSEEIHT, Toulouse, France. A. Mérigaud and J. V. Ringwood are with the Centre for Ocean Energy Research, Department of Electronic Engineering, Maynooth University, Maynooth, Ireland. E-mail: alexis.merigaud.2015@mumail.ie

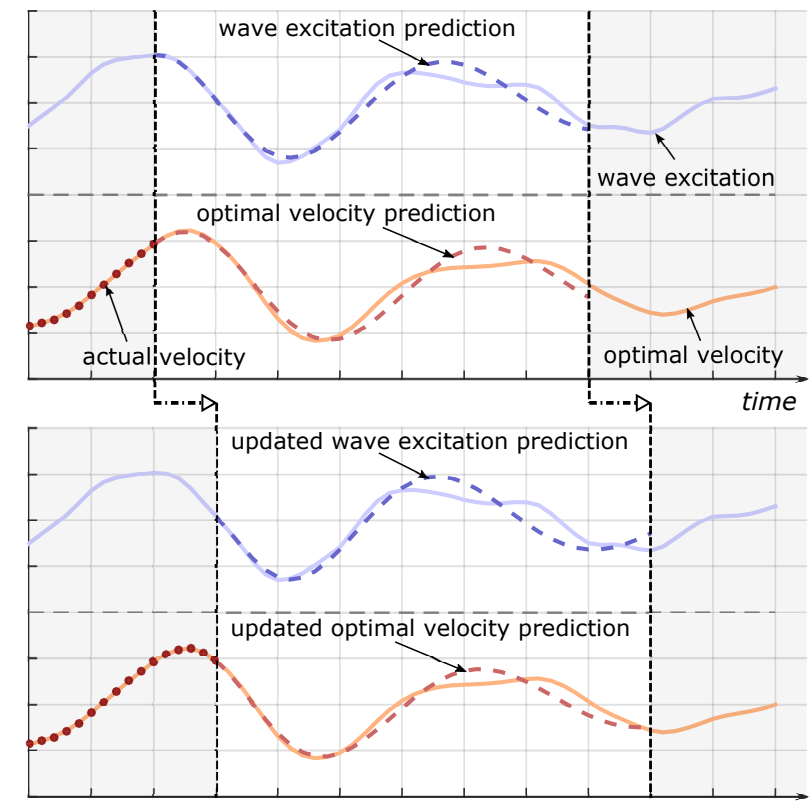

Fig. 1: Receding-horizon WEC control philosophy - optimal velocity trajectory updates at two consecutive time steps. Solid blue (resp. orange): actual wave excitation (resp. optimal WEC trajectory). Dashed blue (resp. red): predicted wave excitation (resp. predicted optimal WEC trajectory). Dotted red: actual trajectory followed by the WEC (assuming perfect tracking).

production is maximised over the finite receding time window, while satisfying the WEC operational constraints. The feedforward set-point velocity trajectory resulting from the optimisation, $v_{f}$, is tracked by a lower-loop controller (LLC), until the next update of the feed-forward trajectory is computed. The corresponding two-level control structure is illustrated in the diagram of Fig. 2. In this paper, however, only the performance of the upper loop (FFC) is investigated.

The accuracy of the reference trajectory, updated by the FFC, is essential in achieving acceptable power absorption, assuming that reasonable tracking performance is achieved by the LLC. In particular, the FFC should accommodate complex WEC dynamics and constraints, while being computable in real-time. The RH configuration (Fig. 1) is also subject to two specific sources of inaccuracies: firstly, the optimisation is carried out over a finite time window only, which implies that not all future wave input information can be taken into account; secondly, the predicted wave excitation signal $e$ is possibly affected by forecast errors.

As stressed in [6], hydrodynamic non-linearities tend to be highlighted under actively controlled conditions compared to, 


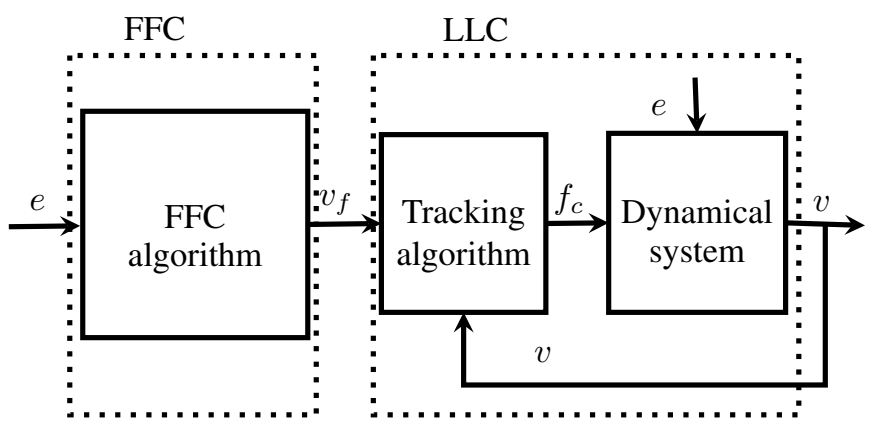

Fig. 2: Simplified diagram of a two-level control structure $v_{f}$ is the feed-forward velocity set-point, $v$ the actual device velocity, $f_{c}$ the PTO control force, $e$ the wave excitation

for example, passive linear damping. In addition, non-linear dynamics may also stem from the characteristics of the PTO machinery or from other physical components, such as the mooring system. Finally, operational constraints must be taken into account. Therefore, the FFC algorithm should be able to handle non-linear effects in the device dynamics, as well as system constraints.

Such non-linear optimisation problems can be difficult to solve (see for example [7]), especially if the length of the RH window results in a large computational load. However, allowing for a discretisation of the continuous-time control problem into a non-linear programming (NLP) problem of finite dimension, pseudospectral (PS) methods have shown some promise, in order to solve the constrained WEC optimal control problem, for linear [8] [3] or non-linear [9] [10] [11] [12] WEC models, while keeping computational requirements relatively low. PS methods are thus interesting candidate tools as FFC algorithms.

In particular, the Fourier PS control formulation - i.e. using harmonic functions as a PS functional basis - shows appealing characteristics in terms of ease of implementation and computational speed [11], [12], and has proven able to compute WEC optimal trajectories under strong non-linearities and constraints [12]. It also constitutes an intuitive choice, given the pseudo-periodic nature of ocean waves. [11] and [12] use a Fourier PS formulation to calculate steady-state optimal control trajectories, in periodic input wave signals (monochromatic in [11], polychromatic in [12]), under various linear and non-linear modelling assumptions and constraints. However, in a $\mathrm{RH}$ implementation, the wave input signal seen by the FFC within the receding window is, in general, not periodic. As a consequence, non-periodic basis functions have been proposed instead of Fourier series, e.g. Lagrange polynomials [9] or Half-Range Chebyshef Fourier functions [3].

Alternatively, this paper explores the pragmatic option of retaining a periodic Fourier approach in a RH implementation, either by simply ignoring the non-periodicity of the finite input wave excitation, or by applying a windowing function to the finite input wave excitation, so as to make the control problem more amenable to a Fourier PS approach. The corresponding requirements in terms of the receding window length are studied.

Retaining a Fourier formulation has another significant advantage with respect to other basis functions: even for long, periodic wave inputs (more than 10 typical wave periods), the Fourier PS framework can be used to compute, at once, the optimal velocity profile over the whole signal, as in [12]. Thus, in this paper, the quality of the feed-forward WEC trajectory, obtained by the FFC within the RH framework, is numerically assessed through a comparison with the actual optimal velocity profile, obtained by taking into account the totality of the wave excitation signal.

In addition, as stressed in Chapter 12 of [13], the effect of wave excitation forecast errors on the performance of $\mathrm{RH}$ controllers has not yet been fully investigated. In this paper, two parallel cases are considered for each set of results: in the first one, the wave excitation forecast is assumed to be perfect over the length of the receding window while, in the second case, future wave elevation values are obtained through a linear predictor, solely based on past measured values of the wave excitation force. Thus, in the latter case, the future wave excitation signal, seen by the controller, contains significant prediction errors.

In summary, this paper describes a methodology to evaluate the quality of the reference trajectory, generated by a FFC algorithm in a RH fashion using a Fourier PS approach, with and without wave forecasting inaccuracies. Other important aspects of a comprehensive RH WEC control framework are beyond the scope of this study: in particular, no constraints are considered; excitation force measurement or estimation is not dealt with; and the LLC tracking is assumed ideal.

The rest of this paper is organised as follows: Section II describes the Fourier PS approach, as well as its implementation as a FFC in a RH set-up. The wave excitation forecasting method is briefly explained in Section III. The numerical case studies (WEC models, sea states, control setup), retained to assess the performance of the proposed FFC, are described in Section IV. The corresponding numerical results are shown and commented in Section V. Finally, conclusions and discussions are presented in Section VI.

\section{FOURIER PSEUDO-SPECTRAL CONTROL}

\section{A. Frequency-domain control formulation}

Let us consider a 1-DoF WEC, whose generalised position is described by means of a scalar coordinate $z$. In the following, it is assumed that Newton's second law, to describe the WEC dynamics, can be written as follows:

$$
g_{l}(z, \dot{z}, \ddot{z})+g_{n l}(z, \dot{z}, \eta)-u(t)-e(t)=0
$$

where

- $g_{l}$ includes inertial terms and forces, which depend on $z$ and its derivatives in a linear way. For example, if all the hydrodynamic forces acting on the device are linearly 
modelled, $g_{l}$ consists of the linear terms of the popular Cummins' equation [14]:

$$
g_{l}(z, \dot{z}, \ddot{z})=\left(\mu+\mu_{\infty}\right) \ddot{z}+\int_{-\infty}^{t} k_{\text {rad }}(t-\tau) \dot{z}(\tau) d \tau+k_{h} z
$$

where $\mu$ is the WEC inertia, $k_{h}$ is a hydrostatic stiffness coefficient, and the radiation forces are computed as the sum of an inertial term $\mu_{\infty} \ddot{z}$, and a convolution product between the past values of the velocity and the radiation impulse response function $k_{\text {rad }}$;

- $g_{n l}$ is an analytical expression, containing the forces which non-linearly depend on $z$, its derivative, and possibly the wave input $\eta$. Obviously, if a part of the hydrodynamic force is non-linearly modelled in $g_{n l}$, the corresponding terms in (2) have to be removed from $g_{l}$;

- The control input $u(t)$ is the force exerted by the PTO system onto the WEC;

- $e(t)$ is an additive wave excitation term, which directly depends on the wave input $\eta$. For example, $e$ may consist of the linear wave excitation or diffraction force.

Let us now consider the control problem for a wave input expanded in a Fourier series as:

$$
\eta(t)=a_{\eta, 0}+\sum_{n=1}^{N-1} a_{\eta, n} \cos \left(\omega_{n} t\right)+b_{\eta, n} \sin \left(\omega_{n} t\right)
$$

where $\omega_{n}=n \Delta \omega, \Delta \omega=2 \pi / T$ is the frequency step, and $T$ is the period of the signal.

The control problem, over $[0 ; T]$, consists of transmitting as much power as possible from the waves to the PTO system:

$$
\begin{aligned}
& \min P(z, u)=\frac{1}{T} \int_{0}^{T} \dot{z}(t) u(t) d t \\
& \text { s.t. } g_{l}(z, \dot{z}, \ddot{z})+g_{n l}(z, \dot{z}, \eta)-u(t)-e(t)=0
\end{aligned}
$$

As in [10], the variables $z$ and $u$ can be approximated using the same basis of harmonic sinusoids used in (3), as:

$$
\begin{aligned}
& z(t) \approx a_{z, 0}+\sum_{n=1}^{N-1} a_{z, n} \cos \left(\omega_{n} t\right)+b_{z, n} \sin \left(\omega_{n} t\right) \\
& u(t) \approx a_{u, 0}+\sum_{n=1}^{N-1} a_{u, n} \cos \left(\omega_{n} t\right)+b_{u, n} \sin \left(\omega_{n} t\right)
\end{aligned}
$$

Let us define $Z$ and $U$ in $\mathbb{R}^{2 N}$, the vectors composed of the Fourier coefficients of $z$ and $u$ respectively. Define a matrix $D \in \mathbb{R}^{(2 N-1) \times(2 N-1)}$ :

$$
D=\left(\begin{array}{ccccccc}
0 & 0 & \cdots & 0 & 0 & \cdots & 0 \\
0 & 0 & \cdots & 0 & \omega_{1} & \cdots & 0 \\
\vdots & \vdots & \ddots & \vdots & \vdots & \ddots & \vdots \\
0 & 0 & \cdots & 0 & 0 & \cdots & \omega_{N-1} \\
0 & -\omega_{1} & \cdots & 0 & 0 & \cdots & 0 \\
\vdots & \vdots & \ddots & \vdots & \vdots & \ddots & \vdots \\
0 & 0 & \cdots & -\omega_{N-1} & 0 & \cdots & 0
\end{array}\right)
$$

The projection of the velocity $\dot{z}$ onto the Fourier basis is obtained from $Z$ as $V=D Z$. As shown in [10], the objective function of (4) can then be simply expressed as

$$
P(Z, U)=\frac{1}{2} Z^{\mathrm{T}} D^{\mathrm{T}} U
$$

Furthermore, inspired by [12], the dynamical equation (1) is also projected onto a Fourier basis, so that $U$ is expressed as a function of the other variables as:

$$
\tilde{U}(Z)=M Z+G_{n l}(Z)-E
$$

where

- The frequency-domain projection of the linear terms $g_{l}$ in (1) is given, in matrix form, as $G_{l}(Z)=M Z$. Typically, when radiation and hydrostatic restoring forces are linearly modelled, the components of $M$ are given as, $\forall i, j \in \llbracket 1 ; N-1 \rrbracket^{2}$,

$$
\begin{aligned}
& M_{1,1}=k_{h} \\
& M_{i+1, j+1}= \begin{cases}-\omega_{i}^{2}\left(\mu+A_{\text {rad }}\left(\omega_{i}\right)\right)+k_{h}, & i=j \\
0, & i \neq j\end{cases} \\
& M_{i+N, j+N}=M_{i+1, j+1} \\
& M_{i+1, j+N}= \begin{cases}\omega_{i} B_{\text {rad }}\left(\omega_{i}\right), & i=j \\
0, & i \neq j\end{cases} \\
& M_{i+N, j+1}=-M_{i+1, j+N}
\end{aligned}
$$

where $A_{\text {rad }}$ and $B_{\text {rad }}$ are the frequency-dependent radiation added mass and damping, respectively.

- $G_{n l}$ represents the Fourier expansion of $g_{n l}$ up to the harmonic of order $N-1$;

- $E$ denotes the Fourier expansion of the additive force term $e$.

Combining with (7), the minimisation problem (4) becomes

$$
\min \tilde{P}(Z):=\frac{1}{2} Z^{\mathrm{T}} D^{\mathrm{T}} \tilde{U}(Z)
$$

which can be efficiently solved using gradient-based optimisation techniques [12]. The solution $Z$ of (9) is the optimal steady-state solution for Problem (4), within the chosen functional space, i.e. amongst the solutions which can be described as Fourier series up to the harmonic of order $N-1$.

Finally, note that the simplifications made here to obtain Eq. (9) require that the control input $u$ is the PTO force itself, and can be explicitly written as a function of the other variables through the dynamical equation in (8). In cases where such a simplification is not possible, the dynamical equation must be expressed as a set of equality constraints, as in [10].

\section{B. Receding-horizon implementation}

Consider a window of length $L$. Given the memory effect of the radiation forces, taking into account both past and future values of the input wave signal, as in [2], is useful for the computation of the updated feed-forward velocity profile. Therefore, the window is defined such that the present time $t$ is in the middle of the window interval, $[t-L / 2 ; t+L / 2]$.

Consider a given time $t_{0}$. Assume that the wave excitation is perfectly known over the window $\left[t_{0}-L / 2 ; t_{0}+L / 2\right]$. 
The input to the FFC algorithm is the windowed excitation force, $e_{w}\left(t_{0}, \tau\right):=e\left(t_{0}+\tau\right), \tau \in[-L / 2 ; L / 2]$, which is used by the FFC algorithm to compute an optimal velocity profile $v_{w}\left(t_{0}, \tau\right), \tau \in[-L / 2 ; L / 2]$. More specifically, the signal $e_{w}\left(t_{0}, \tau\right)$ is developed as a Fourier series, using the frequency step $\Delta \omega=2 \pi / L$, and the optimisation is carried out by solving (9). The solution velocity $V_{w}=D Z_{w}$ is transformed back to the time domain, to obtain $v_{w}\left(t_{0}, \tau\right), \tau \in[-L / 2 ; L / 2]$. $v_{w}\left(t_{0}, \tau\right)$ is only followed by the lower-level control loop until the next update of $v_{w}$, i.e. for $\tau$ close to zero.

Consider a sufficiently long simulation wave signal, of length $T \gg L$. For a simple assessment of the quality of the feed-forward trajectory, $v_{w}(t, \tau)$ is computed at each time step $t \in[0 ; T]$, but only $v_{w}(t, 0)$ is retained, assuming that this value will be ideally followed by the WEC. The overall feed-forward signal is thus given by $v_{f}(t):=v_{w}(t, 0)$, for $t \in[0 ; T]$.

The power obtained by perfectly tracking $v_{f}(t)$ over $[0 ; T]$ is denoted $P_{f}$, and can be calculated as follows:

- Expand $v_{f}(t), t \in[0 ; T]$ in Fourier series, using $\Delta \omega=$ $2 \pi / T$, to obtain $V_{f}$. Calculate $Z_{f}$ such that $D Z_{f}=V_{f}$. From Eq. (8) calculate $U_{f}:=\tilde{U}\left(Z_{f}\right)$, the frequencydomain control force necessary to achieve $V_{f}$;

- Compute $P_{f}=-\frac{1}{2} V_{f}^{\mathrm{T}} U_{f}$

Finally, as mentioned in the introduction, the input excitation $e_{w}(t, \tau)$, in each receding time interval, may be multiplied with a windowing function $w(\tau), \tau \in[-L / 2 ; L / 2]$. Furthermore, when the future wave excitation is predicted, rather than perfectly known, actual values $e_{w}(t, \tau)$ have to be replaced with their predicted counterparts $\hat{e}_{w}(t, \tau)$ for $\tau>0$, as detailed in Section III.

\section{Optimisation over the whole signal}

Consider the simulation signal, of length $T \gg L$. In addition to computing $v_{f}(t)$ (obtained in a $\mathrm{RH}$ fashion as explained in Section II-B), the true optimal velocity $v^{*}(t)$ can also be calculated, off-line, using the simulated wave signal in its entirety (as in [12]). Indeed, the total wave signal is periodic with period $T$. Thus, using the fundamental frequency $\Delta \omega=2 \pi / T$ (but the same cut-off frequency $\omega_{c}=\pi$ as for the RH calculations), the control problem can be parametrised as in Eqs. (3) and (5), and the corresponding Problem (9) is solved.

Of course, the size of Problem (9) for the overall signal is significantly larger than it is for a given RH control problem. However, unlike $v_{f}(t), v^{*}(t)$ needs only be computed off-line, and therefore the computation time for $v^{*}(t)$ is not of primary importance. In each simulated wave signal, $v^{*}(t)$, as well as the resulting optimal power $P^{*}$, provide a point of comparison for $v_{f}(t)$ and $P_{f}$ computed in the same simulated waves. In theory, $v_{f}$ and $P_{f}$ should always be sub-optimal with respect to $v^{*}$ and $P^{*}$.

\section{WAVE EXCITATION FORECASTING}

A cost-effective approach to short-term wave forecasting consists of treating the wave signal as a time series [15], using past measurements at the point of interest (in this case, the
WEC location) to predict the incoming signal. This section shows how the Gaussian description of ocean waves can be used to provide a simple, statistically-optimal predictor, as proposed in [16].

Consider the wave elevation modelled as a stationary, ergodic Gaussian process [17], which is entirely characterised by its mean $(\bar{\eta}=0)$ and its auto-covariance function (ACVF) $R_{\eta \eta}(\tau)$ for $\tau \in \mathbb{R}$ :

$$
R_{\eta \eta}(\tau)=\mathbb{E}[\eta(t) \eta(t+\tau)]
$$

$R_{\eta \eta}(\tau)$ can be computed from the spectral density function (SDF) of the wave process, $S_{\eta \eta}(\omega)$, by means of a Fourier transform, by virtue of the Wiener-Khintchine theorem [17].

Following the definition of a Gaussian random process, any finite, discrete ensemble of wave measurements, taken at various points in time, forms a multivariate, Gaussian random vector. Considering that the wave elevation is sampled, say, every second, define $\mathbf{x}$ as the vector of the last $M$ recorded values (indexed by $m \in \llbracket 1 ; M \rrbracket$ ), and $\mathbf{y}$ the (unknown) vector of the next $N$ wave elevation values (indexed by $n \in \llbracket 1 ; N \rrbracket$ ).

Altogether, the $N+M$ points form a multivariate Gaussian random vector, $\mathbf{v} \in \mathbb{R}^{M+N}$. Its mean is $0_{\mathbb{R}^{M+N}}$ and its variance-covariance matrix, noted $\Sigma_{\mathbf{v v}}$, can be entirely derived from the correlation values between any pair of points in time, or the wave spectrum, i.e. $\Sigma_{\mathbf{v v} i, j}=R_{\eta \eta}\left(t_{j}-t_{i}\right)$.

$\Sigma_{\mathbf{v v}}$ can be written as:

$$
\Sigma_{\mathbf{v v}}=\left(\begin{array}{cc}
\Sigma_{\mathbf{y y}} & \Sigma_{\mathbf{y x}} \\
\Sigma_{\mathbf{x y}} & \Sigma_{\mathbf{x x}}
\end{array}\right)
$$

where $\Sigma_{\mathbf{y} \mathbf{x}}=\Sigma_{\mathbf{x y}}^{\mathrm{T}}$. Using $\mu_{\mathbf{x}}=\mu_{\mathbf{y}}=0$, the conditional distribution of $\mathbf{y} \mid \mathbf{x}$ is multivariate Gaussian (see for example [18]) with mean:

$$
\mu_{\mathbf{y} \mid \mathbf{x}}=\Sigma_{\mathbf{y} \mathbf{x}} \Sigma_{\mathbf{x} \mathbf{x}}^{-1} \mathbf{x}
$$

and variance:

$$
\Sigma_{\mathbf{y} \mid \mathbf{x}}=\Sigma_{\mathbf{y} \mathbf{y}}-\Sigma_{\mathbf{y} \mathbf{x}} \Sigma_{\mathbf{x x}}^{-1} \Sigma_{\mathbf{x y}}
$$

The best predictor of $\mathbf{y}$, in a least mean-square sense, is given as

$$
\hat{\mathbf{y}}=\mu_{\mathbf{y} \mid \mathbf{x}}=\Sigma_{\mathbf{y} \mathbf{x}} \Sigma_{\mathbf{x x}}^{-1} \mathbf{x}
$$

It is convenient to define the prediction matrix, $Q:=$ $\Sigma_{\mathbf{y x}} \Sigma_{\mathbf{x x}}^{-1}$, which maps $M$ measured values to $N$ predicted values. The mean-square prediction error $\epsilon^{2}(h)$, for each prediction horizon $h$, is given by the diagonal terms of $\Sigma_{\mathbf{y} \mid \mathbf{x}}$. For a given order $M$, any other forecasting method is suboptimal with respect to the law derived in (14), to evaluate the $N$ predicted points.

The prediction matrix $Q$ needs only be updated as the wave condition evolves significantly (e.g. every 30 minutes), and the only operation carried out in real-time is the multiplication $\hat{\mathbf{y}}=Q \mathbf{x}$.

In the case study presented in this paper, it is assumed that the wave excitation torque, rather than the wave elevation itself, is predicted, assuming that the torque can be measured or estimated in real-time [19], [20]. Therefore, in the above analysis, the wave elevation $\operatorname{SDF} S_{\eta}(\omega)$ is simply replaced with the wave excitation torque $\mathrm{SDF}$, obtained as $S_{e}(\omega)=$ $S_{\eta}(\omega)\left|H_{\eta e}(\omega)\right|^{2}$, where $H_{\eta e}(\omega)$ is the linear wave-to-torque 


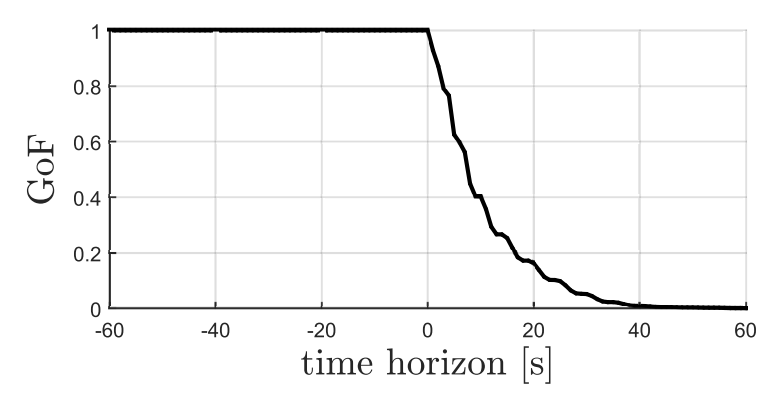

Fig. 3: GoF of the excitation torque prediction, JONSWAP spectrum $\left(H_{s}=2 \mathrm{~m}, T_{p}=10 \mathrm{~s}\right)$

transfer function for the flap-type WEC, computed with the hydrodynamic software WAMIT ${ }^{1}$. The WEC is introduced in more detail in Section IV-A.

Orders $M$ higher than 200 (for a time step of 1 second) were found not to bring any significant improvement in the accuracy of the predictions. Therefore, an order of 200 was retained. Fig. 3 shows the corresponding goodness of fit (GoF) of the excitation torque prediction, computed as

$$
G(h)=1-\sqrt{\frac{\epsilon^{2}(h)}{\mathbb{E}\left[e^{2}\right]}}
$$

for a JONSWAP spectrum with $H_{s}=2 \mathrm{~m}$ and $T_{p}=10 \mathrm{~s}$, and where $\mathbb{E}\left[e^{2}\right]$ represents the variance of the excitation torque process.

\section{NUMERICAL CASE STUDIES}

\section{A. WEC model}

A flap-type wave energy converter, subject to a quadratic viscous drag force, is chosen. The hydrodynamic model is identical to the one used in [10]. The only degree of freedom is the WEC angular position. The radiation and excitation forces are represented linearly. The frequency-domain coefficients $A_{\text {rad }}(\omega), B_{\text {rad }}(\omega)$ and $E(\omega)$ are computed with the hydrodynamic software WAMIT ${ }^{2}$. A linear model is also considered, by simply removing the quadratic drag term.

\section{B. Numerical set-up}

The sea states considered are expressed by JONSWAP [21] spectra with typical $H_{s}$ and $T_{p}$ values $\left(T_{p} \in[6 ; 14]\right.$ and $\left.H_{s} \in[1 ; 4]\right)$. However, unless specified otherwise, the numerical results are plotted for $H_{s}=2 m$ and $T_{p}=10 s$. The prediction matrix $Q$ (see Section III) is computed for each sea spectrum.

The FFC algorithm performance is assessed over wave signals of length $T=300 \mathrm{~s}$. The time step (both for $t$ and $\tau)$ is 1 second, which corresponds to a cut-off frequency $\omega_{c}=\pi \mathrm{rad} / \mathrm{s}$. As explained in Section II-B, the feed-forward velocity trajectory $v_{f}$ is updated every second by the FFC algorithm, using $L$ data points (the fundamental frequency is $\left.\omega_{1}=2 \pi / T_{w}\right)$. In contrast, the optimal velocity $v^{*}(t)$ is

\footnotetext{
${ }^{1}$ http://www.wamit.com/

${ }^{2}$ http://www.wamit.com/
}

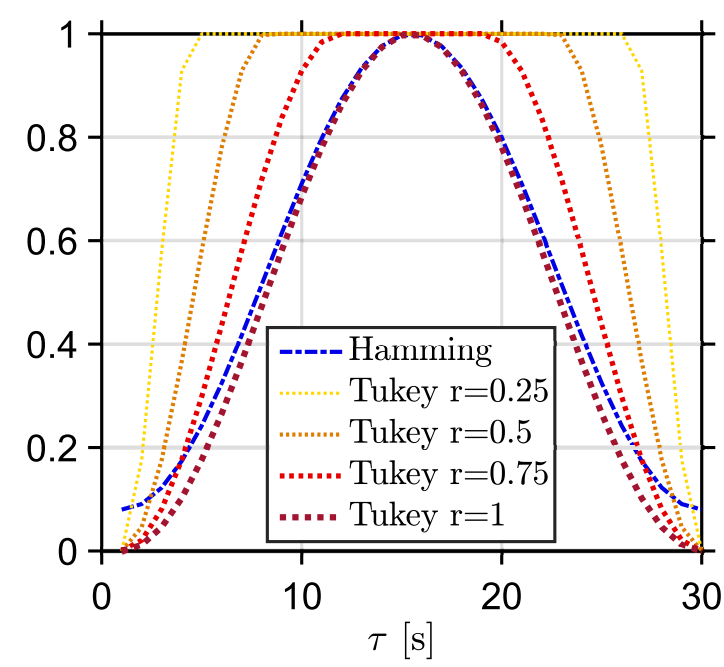

Fig. 4: Various windowing functions

computed by considering the totality of the wave signal, as explained in Section II-C.

The non-linear control problems (9), in both the RH implementation and for the calculation of $v^{*}$, are solved using a trust-region algorithm (readily implemented in Matlab within the fminunc solver ${ }^{3}$ ).

Tukey windows [22] are investigated, in order to make the finite-length wave excitation signal more suitable for a control framework involving periodic basis functions. Tukey windows leave the signal unchanged in its middle, over a length defined by the parameter $r \in[0 ; 1]$, as illustrated in Fig. 4, and thus provide an interesting family of windows to investigate. A Hamming window, which does not exactly bring the signal to zero at the ends, is also investigated. Note that the absence of windowing function can also be seen as the default application of a rectangular window:

\section{Simulation procedure}

To assist the reader in connecting the different sections of this paper, the experimental procedure for a given simulated wave signal (here, assuming that the wave excitation is predicted, and that the RH signal is windowed through a window function $w(\tau)$ ) is summarised as follows:

1- From excitation force spectrum $S_{e}(\omega)$, randomly generate simulation wave excitation signal $e(t)$ with period $T=$ $300 \mathrm{~s}$.

2- Off-line prediction matrix calculation: From $S_{e}(\omega)$, compute the matrix $Q$ following the procedure given in Section III.

3- Online calculation of $v_{f}$ : With $\Delta t=1 \mathrm{~s}$, and for $t=$ $\Delta t, 2 \Delta t, \ldots N_{s} \Delta t=T$ :

a) Forecasting: Define measured vector $\mathrm{x}$ with components $\mathbf{x}_{i, i=0 \ldots 200}=e(t-i \Delta t)$. Calculate $\mathbf{y}=Q \mathbf{x}$ with $\mathbf{y}_{i, i=1 \ldots L / 2}=\hat{e}(t+i \Delta t)$, the vector of predicted values. See also Section III.

\footnotetext{
${ }^{3}$ https://uk.mathworks.com/help/optim/
} 


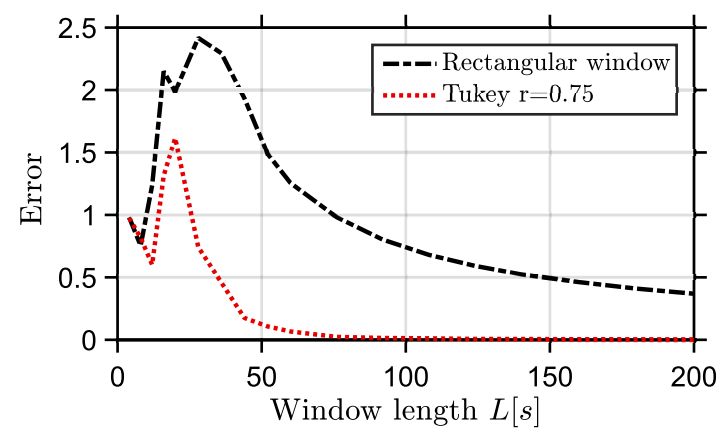

Fig. 5: $\epsilon_{\text {RMS }}$ between $v_{f}$ and $v^{*}$ - linear WEC model

b) Define $\tau_{i}=i \Delta t$, and

$$
e_{w}\left(t, \tau_{i}\right):=\left\{\begin{array}{l}
e\left(t+\tau_{i}\right), \tau_{i} \in[-L / 2 ; 0] \\
\hat{e}\left(t+\tau_{i}\right), \tau_{i} \in[1 ; L / 2]
\end{array}\right.
$$

c) Windowing: Compute $e_{w}^{\prime}\left(t, \tau_{i}\right)=w\left(\tau_{i}\right) e_{w}\left(t, \tau_{i}\right)$.

d) Develop $e_{w}^{\prime}(t, \tau)$ in Fourier series, using $\Delta \omega=$ $2 \pi / L$, and solve (9) to obtain $v_{w}^{*}(t, \tau), \tau \in$ $[-L / 2 ; L / 2]$. See also Sections II-A and II-B.

e) Define $v_{f}(t):=v_{w}^{*}(t, 0)$

4- From $v_{f}(t), t \in[0 ; T]$, calculate $P_{f}$ as explained in Section II-B.

5- Off-line control calculation: Develop $e(t)$ in Fourier series using $\Delta \omega=2 \pi / T$, and solve (9) to obtain $v^{*}(t)$ and $P^{*}$. See also Sections II-A and II-C.

\section{NUMERICAL RESULtS}

\section{A. Assuming perfect knowledge of the future wave excitation}

The optimal velocity, $v^{*}(t)$, and the feed-forward velocity reference, $v_{f}$, are compared through the root-mean-square error, computed as:

$$
\epsilon_{\mathrm{RMS}}=\sqrt{\frac{\sum_{t=0}^{T}\left(v_{f}(t)-v^{*}(t)\right)^{2}}{\sum_{t=0}^{T} v(t)^{* 2}}}
$$

Assuming perfect knowledge of the wave signal over the whole window length, and considering a linear WEC model, Fig. 5 shows $\epsilon_{\text {RMS }}$ for different window lengths, using different windowing functions. When no windowing function is applied, i.e. assuming a default rectangular window, the FFC is clearly unable to compute a velocity trajectory close to the optimal one, even for large $L$ values. Accordingly, the power absorption performance is poor, $P_{f}$ taking positive values only for $L \geq 150$ s (Fig. 6).

However, the results can be significantly improved by windowing the FFC input signal $e_{w}$, such as described in Section IV-B (Figs. 5 and 6). The Tukey window with $r=0.75$ is found to be the best choice amongst the studied options, allowing $P_{f}$ to reach more than $99 \%$ of $P^{*}$ with $L=56 \mathrm{~s}$.

In contrast, with the non-linear WEC model including a quadratic viscous drag force, the error function is relatively small (Fig.7) and $P_{f}$ is close to $P^{*}$, even for relatively short windows (Fig. 8). The quadratic damping term seems to reduce the sensitivity of the FFC performance to the window length.

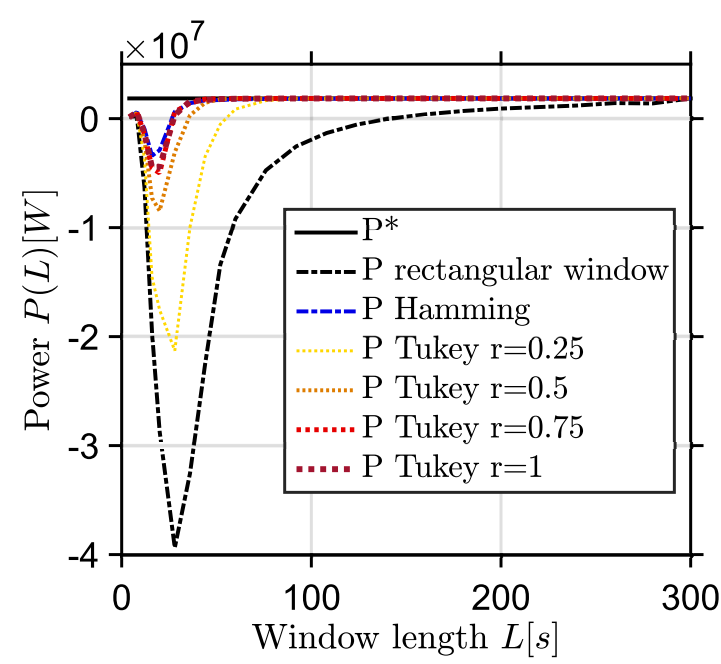

Fig. 6: $P_{f}$ resulting from tracking $v_{f}$, for different window lengths and windowing functions - linear WEC model

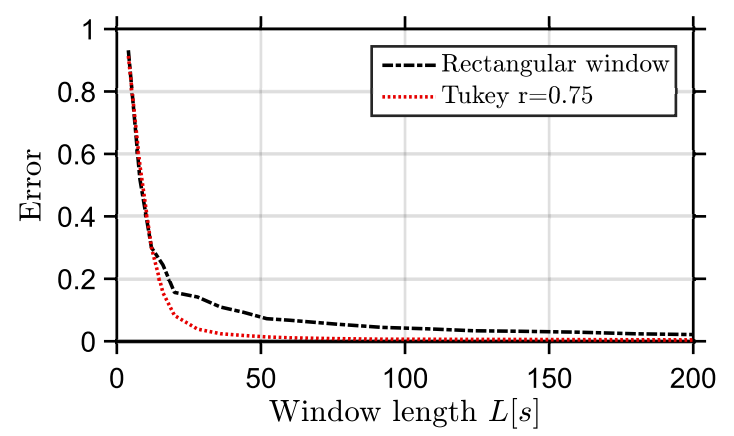

Fig. 7: $\epsilon_{\mathrm{RMS}}$ between $v_{f}$ and $v^{*}$ - non-linear WEC model

Indeed, since the damping term reduces the order of magnitude of the optimal velocity, the relative impact of the radiation terms, and the associated memory effect, are also reduced.

Accordingly, the improvement brought by applying window functions, in the presence of viscous damping, is less significant. However, as for the linear case, the Tukey window with $r=0.75$ is found to be the best option, with $P_{f}$ exceeding $99 \%$ of $P^{*}$ with $L=22 s$ only.

\section{B. With (imperfect) wave excitation forecasts}

Instead of assuming perfect knowledge of the wave excitation, forecasts are now carried out as explained in Section III so that, within each window, the first half of the signal is assumed to be perfectly known, while the second half consists of predicted values, as illustrated in Fig. 9. For the sake of conciseness, results presented further in this subsection are all obtained by adopting the Tukey window with $r=0.75$.

For the linear device, Figs. 11 and 10 show that forecast errors have a modest impact on the quality of the feed-forward trajectory $v_{f}: 99 \%$ of $P^{*}$ can still be achieved with $L=56 \mathrm{~s}$.

Results are similar for the non-linear device, although $v_{f}$ seems to contain some residual error, even for large $L$ values (Fig. 12), which can be attributed to the forecast errors. However, the power production is not significantly adversely affected (Fig. 13). 


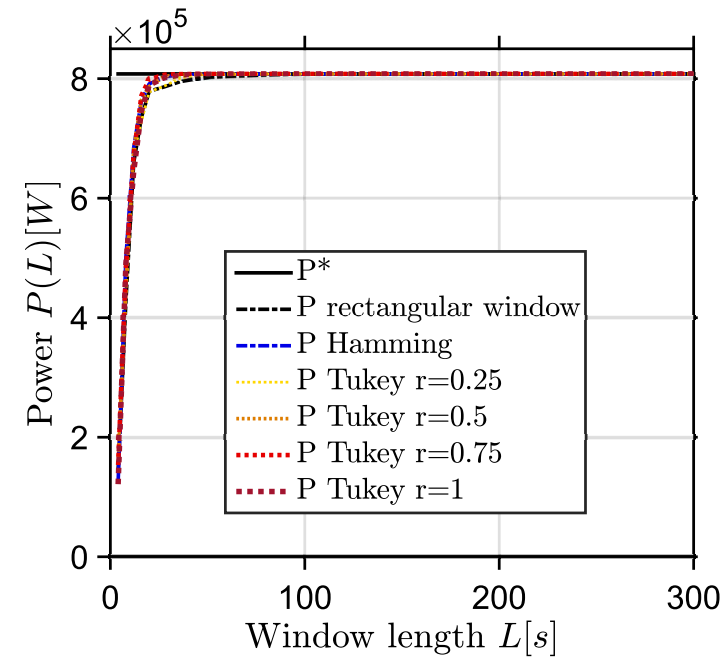

Fig. 8: $P_{f}$ resulting from tracking $v_{f}$, for different window lengths and windowing functions - non-linear WEC model

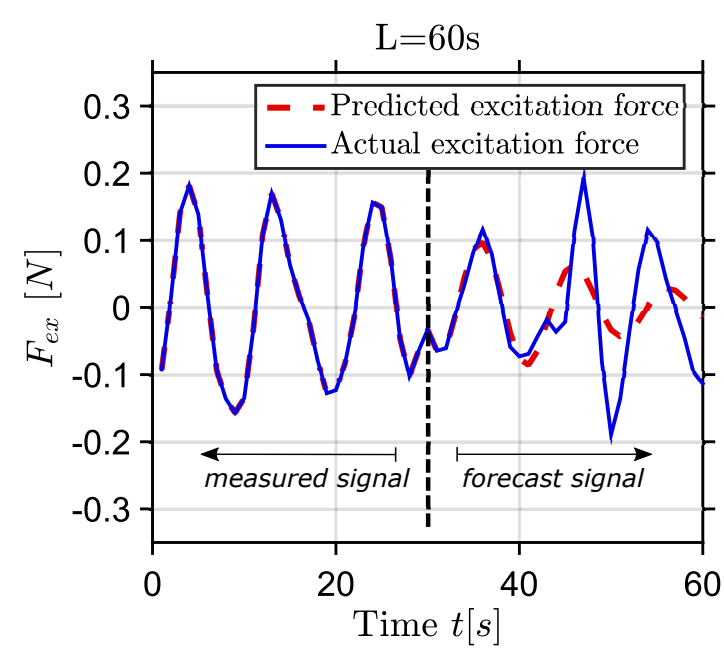

Fig. 9: Example of input wave excitation signal within a receding time interval - actual $v s$ predicted values

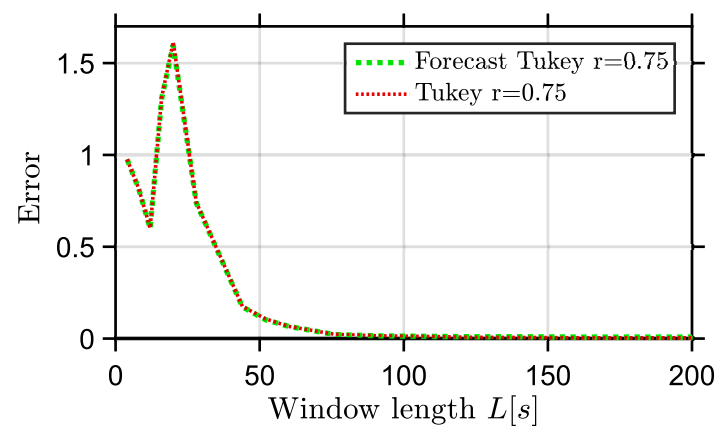

Fig. 10: RMSE between $v_{f}$ and $v^{*}$, with actual and forecast wave excitation - linear WEC model

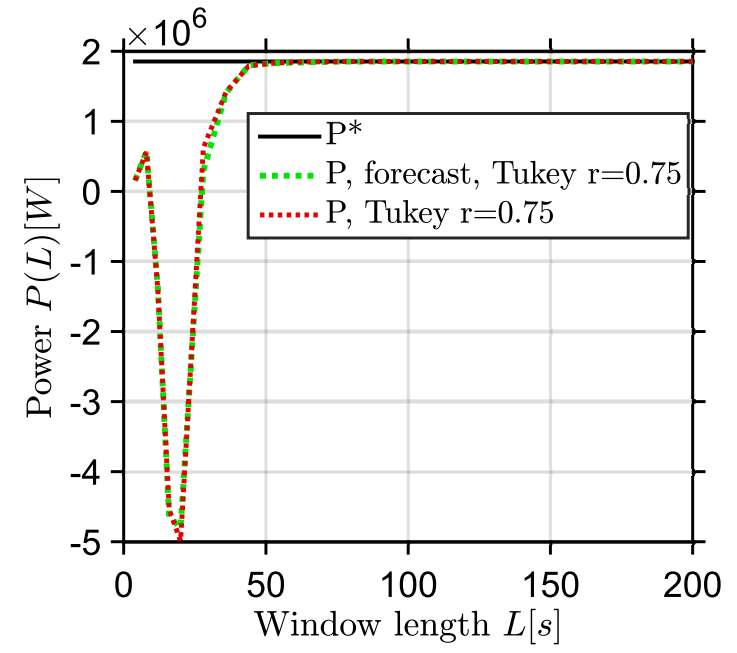

Fig. 11: $P_{f}$ resulting from tracking $v_{f}$, with actual and forecast wave excitation - linear WEC model

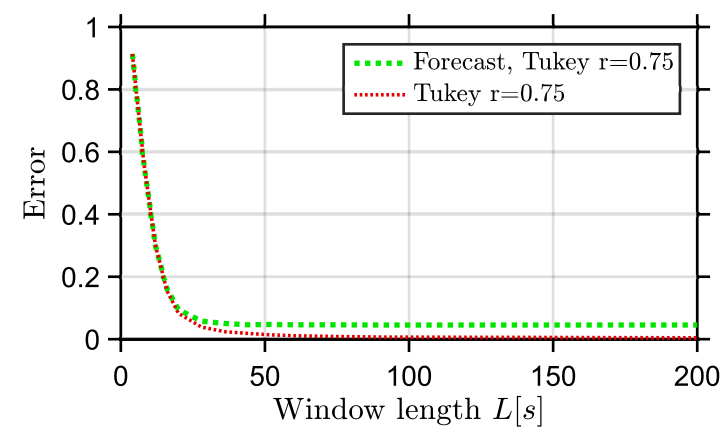

Fig. 12: RMSE between $v_{f}$ and $v^{*}$, with actual and forecast wave excitation - non-linear WEC model

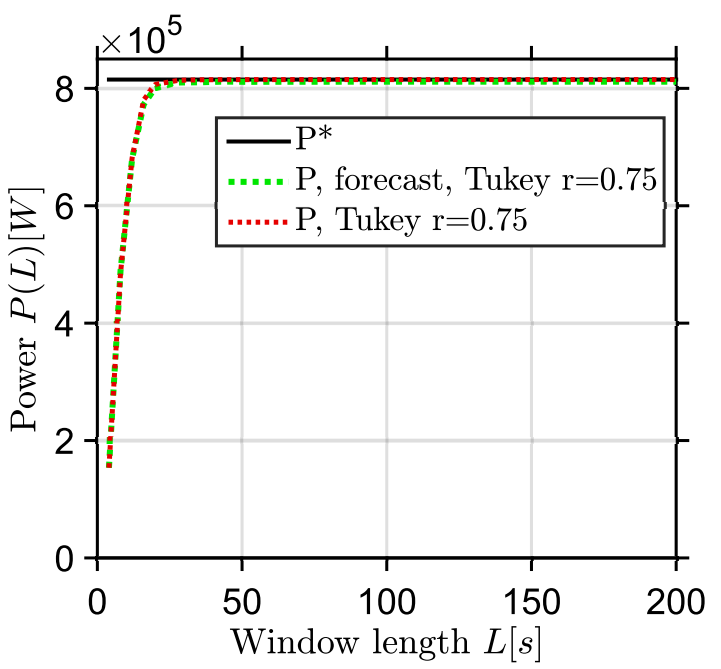

Fig. 13: $P_{f}$ resulting from tracking $v_{f}$, with actual and forecast wave excitation - non-linear WEC model 
TABLE I: $L$ [s] yielding $99 \%$ of $P^{*}$, in a range of JONSWAP spectra (linear WEC model)

\begin{tabular}{lllll}
\multicolumn{5}{c}{$T_{p}[s]$} \\
6 & 8 & 10 & 12 & 14 \\
\hline $58 \mathrm{~s}$ & $57 \mathrm{~s}$ & $56 \mathrm{~s}$ & $52 \mathrm{~s}$ & $51 \mathrm{~s}$
\end{tabular}

TABLE II: $L$ [s] yielding $99 \%$ of $P^{*}$, in a range of JONSWAP spectra (non-linear WEC model)

\begin{tabular}{lllllll} 
& & \multicolumn{5}{c}{$T_{p}[s]$} \\
& & 6 & 8 & 10 & 12 & 14 \\
\hline \multirow{3}{*}{$H_{s}[\mathrm{~m}]$} & 1 & $30 \mathrm{~s}$ & $26 \mathrm{~s}$ & $25 \mathrm{~s}$ & $24 \mathrm{~s}$ & $23 \mathrm{~s}$ \\
& 2 & $28 \mathrm{~s}$ & $25 \mathrm{~s}$ & $22 \mathrm{~s}$ & $21 \mathrm{~s}$ & $19 \mathrm{~s}$ \\
& 3 & & $22 \mathrm{~s}$ & $20 \mathrm{~s}$ & $19 \mathrm{~s}$ & $18 \mathrm{~s}$ \\
& 4 & & & $19 \mathrm{~s}$ & $19 \mathrm{~s}$ & $19 \mathrm{~s}$
\end{tabular}

For the sake of brevity, only the results obtained using a JONSWAP spectrum with $H_{s}=2 m$ and $T_{p}=10 s$ are presented in this section. However, conclusions remain similar with other values for $H_{s}$ and $T_{p}$. Tables I and II show that, regardless of the sea state, $99 \%$ of $P^{*}$ can be achieved, with $L \in[51: 58]$ for the linear WEC model, and $L \in[19: 30]$ for the non-linear WEC model.

Overall, in spite of significant forecast errors, it seems possible to achieve a $P_{f}$ close to $P^{*}$, by properly choosing the receding window length, and by applying appropriate windowing functions.

\section{CONCLUSIONS AND DISCUSSIONS}

\section{A. Conclusions}

The results presented in this paper clearly demonstrate the applicability of PS methods based on periodic functions, for the computation of the feed-forward velocity reference within a RH control scheme for WEC power maximisation, using both linear and non-linear WEC models. In the proposed set-up, the receding window is chosen in such a way that the present time (where the computed velocity reference will actually be tracked) is in its centre. The receding window should be long enough, so that the accuracy of the feedforward velocity trajectory is ensured, but not so long as to make the optimisation problem intractable in real-time.

For a linear WEC model, the window length requirements, necessary to obtain a feed-forward velocity reference with sufficient quality, can be large (more than 200s). However, applying simple windowing functions to the input wave excitation signal, seen by the FFC algorithm, can dramatically reduce the window length requirements, making it possible to achieve quasi-optimal performance - within $99 \%$ of the optimal power - using window lengths smaller than 60s.

When a non-linear (quadratic) viscous loss term is included in the WEC model, the window lengths, necessary to achieve quasi-optimal performance, are significantly smaller (less than 30s) although the achievable power extraction is obviously smaller than for the linear WEC model. In the non-linear case, the benefits of using windowing functions are thus relatively modest.

A simple technique for wave excitation forecasting is also proposed, assuming that the sea state is known, and that the wave excitation torque experienced by the WEC can be measured or estimated in real time. Although the prediction errors introduced by the simple forecasting algorithm are relatively large, they do not seem to significantly affect the quality of the feed-forward velocity calculation, irrespective of whether the WEC model is linear or non-linear. These findings suggest that the required window lengths are not so much because accurate forecasts are necessary a long time ahead, but rather because the window must be sufficient to allow for accurate Fourier calculations, as also suggested by other researchers [23]. Besides, although errors may appear large, low-frequency wave components, also the most important for energy absorption, are better predicted than their higherfrequency counterparts, which can also explain the relatively good performance of the receding-horizon calculations in spite of the prediction inaccuracies.

\section{B. Limitations and further developments}

The set-up, described in this study, allows for assessing the accuracy of the feed-forward velocity calculations, with respect to the optimal trajectory. However, the short-term dynamics of the lower-level control loop, and their interaction with the update rate of the FFC algorithm, are not considered.

Furthermore, the WEC model, used as a case study throughout this work, is not subject to any operational constraint. The satisfaction of constraints by the RH scheme, while achieving satisfactory power absorption, needs to be investigated. In particular, the presence of constraints may positively or negatively influence the window length and prediction accuracy requirements. More generally, the applicability of the proposed methodology to other WEC models should also be examined on a case-by-case basis.

Issues related to the computation time have not been discussed, in particular for non-linear control calculations, which are the most computationally demanding. However, with a Matlab implementation and the $3.50 \mathrm{GHz}, 8$-core Intel ${ }^{\circledR}$ processor used in this paper, the non-linear control problems, for each windowed signal, were solved by the Fourier PS algorithm (using fminunc) in less than $10 \mathrm{~ms}$ (for a 30-s window length, the most unfavourable case), which is well within the requirements of real-time implementation. For comparison, the computation time reported in [24], for a linear WEC model, without constraints, is approximately $5 \mathrm{~ms}$ using the Half-range Chebyshef Fourier basis (for a 20-s window length). Furthermore, unlike the power level obtained in this paper, which always reaches approximately $100 \%$ of the theoretical maximum for sufficient window lengths, the power obtained in [24] remains sub-optimal. A detailed comparison of the proposed method, with non-periodic PS methods and MPC algorithms, both in terms of power absorption and computation time, similar to [24], is pertinent. The other tasks involved at each update of the reference trajectory (forecasting, windowing) have negligible computational costs, compared to the non-linear control problem. In particular, the prediction method used in this paper is linear, and its coefficients need only be updated as the wave condition evolves. Hence the only real-time operation is a simple matrix multiplication.

Finally, the predictor proposed in this paper assumes perfect measurements of the excitation torque signal, ignoring 
the problem of physical measurements or requirement for estimation techniques. In addition, realistic wave spectra are often less narrow-banded than JONSWAP spectra, which, as documented in [16], can negatively affect the forecast quality.

In view of the above considerations, a more practicallyoriented implementation of the proposed controller, comprising a velocity tracking loop, constrained dynamics, estimation of the excitation signal, as well as a closer examination of computational time requirements, will be investigated by the authors in a future study.

\section{ACKNOWLEDGMENT}

This paper is based upon work supported by Science Foundation Ireland under Grant No. 12/RC/2302 for the Marine Renewable Ireland (MaREI) centre.

\section{REFERENCES}

[1] J. V. Ringwood, G. Bacelli, and F. Fusco, "Energy-maximizing control of wave-energy converters: the development of control system technology to optimize their operation," IEEE Control Systems, vol. 34, no. 5, pp. 30-55, 2014.

[2] F. Fusco and J. V. Ringwood, "A study of the prediction requirements in real-time control of wave energy converters," IEEE Transactions on Sustainable Energy, vol. 3, no. 1, pp. 176-184, 2012.

[3] R. Genest and J. V. Ringwood, "Receding horizon pseudospectral control for energy maximization with application to wave energy devices," IEEE Transactions on Control Systems Technology, vol. 25, no. 1, pp. 29-38, 2017.

[4] N. Faedo, S. Olaya, and J. V. Ringwood, "Optimal control, MPC and MPC-like algorithms for wave energy systems: An overview," IFAC Journal of Systems and Control, 2017.

[5] F. Fusco and J. V. Ringwood, "Hierarchical robust control of oscillating wave energy converters with uncertain dynamics," IEEE Transactions on Sustainable Energy, vol. 5, no. 3, pp. 958-966, 2014.

[6] M. Penalba Retes, A. Mérigaud, J.-C. Gilloteaux, and J. Ringwood, "Nonlinear Froude-Krylov force modelling for two heaving wave energy point absorbers," in Proceedings of the 11th European Wave and Tidal Energy Conference, Nantes, France, 2015.

[7] M. Richter, M. E. Magana, O. Sawodny, and T. K. Brekken, "Nonlinear model predictive control of a point absorber wave energy converter," IEEE Transactions on Sustainable Energy, vol. 4, no. 1, pp. 118-126, 2013.

[8] G. Bacelli and J. V. Ringwood, "Numerical optimal control of wave energy converters," IEEE Transactions on Sustainable Energy, vol. 6, no. 2, pp. 294-302, 2015.

[9] G. Li, "Predictive control of a wave energy converter with wave prediction using differential flatness," in Proceedings of the IEEE 54th Annual Conference on Decision and Control (CDC). IEEE, 2015, pp. 3230-3235.

[10] G. Bacelli and J. V. Ringwood, "Nonlinear optimal wave energy converter control with application to a flap-type device," in Proceedings of the 19th IFAC World Congress, vol. 47, no. 3. Elsevier, 2014, pp. 7696-7701.

[11] G. Bacelli, R. Genest, and J. V. Ringwood, "Nonlinear control of flaptype wave energy converter with a non-ideal power take-off system," Annual Reviews in Control, vol. 40, pp. 116-126, 2015.

[12] A. Mérigaud and J. V. Ringwood, "Optimal trajectories, nonlinear models and constraints in wave energy device control," in Proceedings of the 20th IFAC world congress 2017, Toulouse, France, vol. 50, 2017, pp. $15645-15650$.

[13] U. A. Korde and J. V. Ringwood, Hydrodynamic Control of Wave Energy Devices. Cambridge University Press, 2016.

[14] W. E. Cummins, "The impulse response function and ship motions," in Schiffstechnik, 1962, no. 9, pp. 101-109.

[15] F. Fusco and J. V. Ringwood, "Short-term wave forecasting for real-time control of wave energy converters," IEEE Transactions on Sustainable Energy, vol. 1, no. 2, pp. 99-106, 2010.

[16] A. Mérigaud and J. V. Ringwood, "Incorporating ocean wave spectrum information in short-term free-surface elevation forecasting," IEEE Journal of Oceanic Engineering, Accepted Jan. 2018.
[17] M. K. Ochi, Ocean Waves: The Stochastic Approach. Cambridge University Press, 2005

[18] M. L. Eaton, "Multivariate statistics: A vector space approach," Lecture Notes-Monograph Series, vol. 53, pp. i-512, 2007. [Online]. Available: http://www.jstor.org/stable/20461449

[19] O. Abdelkhalik, S. Zou, G. Bacelli, R. D. Robinett, D. G. Wilson, and R. G. Coe, "Estimation of excitation force on wave energy converters using pressure measurements for feedback control," in Proceedings of the OCEANS 2016 MTS/IEEE conference, Monterey. IEEE, 2016, pp. $1-6$.

[20] M. Abdelrahman, R. Patton, B. Guo, and J. Lan, "Estimation of wave excitation force for wave energy converters," in Proceedings of the $3 \mathrm{rd}$ Conference on Control and Fault-Tolerant Systems (SysTol). IEEE, 2016, pp. 654-659.

[21] K. Hasselmann, T. Barnett, E. Bouws, H. Carlson, D. Cartwright, K. Enke, J. Ewing, H. Gienapp, D. Hasselmann, P. Kruseman et al., "Measurements of wind-wave growth and swell decay during the joint north sea wave project (JONSWAP)," Deutches Hydrographisches Institut, Tech. Rep., 1973.

[22] F. J. Harris, "On the use of windows for harmonic analysis with the discrete Fourier transform," Proceedings of the IEEE, vol. 66, no. 1, pp. $51-83,1978$.

[23] G. Li, G. Weiss, M. Mueller, S. Townley, and M. R. Belmont, "Wave energy converter control by wave prediction and dynamic programming," Renewable Energy, vol. 48, pp. 392-403, 2012.

[24] R. Genest and J. V. Ringwood, "A critical comparison of modelpredictive and pseudospectral control for wave energy devices," Journal of Ocean Engineering and Marine Energy, vol. 2, no. 4, pp. 485-499, 2016.

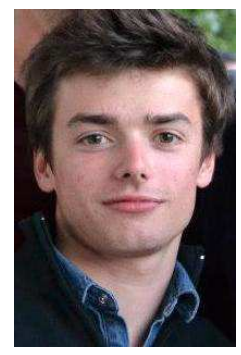

Clément Auger graduated from ENSEEIHT, a French engineering school in Toulouse, France, in 2018. He is studying water and environmental sciences. During the summer 2017, he worked at the Center for Ocean Energy Research, NUI Maynooth, Ireland for a 12 week internship.

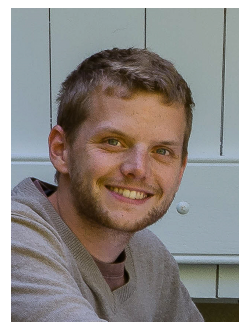

Alexis Mérigaud started his PhD in January 2015 at COER (Centre for Ocean Energy Research) in Maynooth University, Ireland. He is working on numerical simulation and control for linear and nonlinear wave energy converter models, as well as realtime wave forecasting. He obtained his engineering degree in 2012 at ENSTA in Paris where he specialised in Energy management and economics. He then studied Public administration for two years at Sciences Po Paris. His previous experience in the field of energy includes economic modelling, as well as technical regulatory aspects, of energy networks and markets.

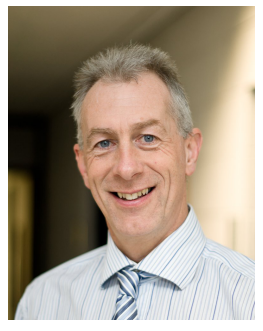

John Ringwood received the Diploma in Electrical Engineering from Dublin Institute of Technology and the $\mathrm{PhD}$ (in Control Systems) from Strathclyde University, Scotland in 1981 and 1985 respectively. $\mathrm{He}$ is currently Professor of Electronic Engineering at Maynooth University Ireland and Director of the Centre for Ocean Energy Research there. He was Head of the Electronic Engineering Department at NUI Maynooth from 2000 until 2005, developing the Department from a greenfield site. John's research interests cover time series modelling, wave energy, control of plasma processes and biomedical engineering. He is a Chartered Engineer and a Fellow of the Institution of Engineers of Ireland. 